\title{
FLNC pathogenic variants in patients with cardiomyopathies Prevalence and genotype-phenotype correlations
}

\section{Running Title : FLNC variants genotype-phenotype correlation}

Flavie Ader1,2,3, Pascal De Groote ${ }^{4}$, Patricia Réant5, Caroline Rooryck-Thambo6, Delphine Dupin-Deguine ${ }^{7}$, Caroline Rambaud ${ }^{8}$, Diala Khraiche ${ }^{9}$, Claire Perret ${ }^{2}$, Jean Francois Pruny ${ }^{10}$, Michèle Mathieu Dramard ${ }^{11}$, Marion Gérard12, Yann Troadec ${ }^{12}$, Laurent Gouya ${ }^{13}$, Xavier Jeunemaitre ${ }^{14}$, Lionel Van Maldergem ${ }^{15}$, Albert Hagège ${ }^{16}$, Eric Villard², Philippe Charron², 10, Pascale Richard11,2, 10.

Conflict of interest statement: none declared for each author

1. APHP, UF Cardiogénétique et Myogénétique, Service de Biochimie Métabolique, Hôpitaux Universitaires de la Pitié- Salpêtrière- Charles Foix, 47-83 Bd de l'Hôpital, Paris, France

2. Sorbonne Université, UPMC Univ. Paris 06, INSERM, UMR_S 1166 and ICAN Institute for Cardiometabolism and Nutrition, Paris, France

3. Université Paris Descartes, Faculté de Pharmacie, 4 avenue de l'Observatoire, 75270 Paris, France

4. Pôle Cardio-Vasculaire et Pulmonaire, CHRU de Lille - Hôpital Albert Calmette, Boulevard du Pr Jules Leclercq, Lille, France

5. Service de Cardiologie, CHU de Bordeaux, Université de Bordeaux, France

6. CHU Bordeaux, Service de Génétique Médicale, F-33000 Bordeaux, France

7. Service de génétique médicale, et service d'otoneurochirurgie, $\mathrm{CHU}$ de Toulouse - Hôpital Purpan, Place du Docteur Baylac, Toulouse, France

8. Service Médecine Légale, Hôpital Raymond Poincaré, Garches, France

9. APHP, Service de Cardiologie, Hôpital Necker, Paris, France

10. APHP, Centre de référence pour les maladies cardiaques héréditaires, Hôpitaux Universitaires Pitié-Salpêtrière, Paris

11. CHU Amiens Picardie, Service de Génétique Clinique, Amiens, France

12. CHU Caen, Service de Génétique Médicale, Caen, France

13. APHP, Service de Génétique Médicale, CHU Bichat-Claude Bernard, Paris, France

14. APHP, Service de génétique, Hôpital Européen Georges Pompidou, Paris, France 
15. Centre de génétique humaine, Université de Franche Comté, Besançon, France

16. APHP, Service de Cardiologie, Hôpital Européen Georges Pompidou, Paris, France

\section{Acknowledgements}

Lab team is warmly acknowledged for the technical realization of this project. We would like to thank patients and their family members for their cooperation. This work was supported by Assistance Publique Hôpitaux de Paris (APHP), Fondation pour la Recherche Médicale (FRM), and the Association of patients « Ligue contre la Cardiomyopathie ».

\section{Data Availability Statement}

The data that support the findings of this study are available from the corresponding author upon reasonable request. 


\section{Abstract}

Pathogenic variants in FLNC encoding filamin C have been firstly reported to cause myopathies, and were recently linked to isolated cardiac phenotypes. Our aim was to estimate the prevalence of FLNC pathogenic variants in subtypes of cardiomyopathies and to study the relations between phenotype and genotype.

DNAs from a cohort of 1150 unrelated index-patients with an isolated cardiomyopathy (700 hypertrophic, 300 dilated, 50 restrictive cardiomyopathies, and 100 left ventricle non-compactions) have been sequenced on a custom panel of 51 cardiomyopathy disease-causing genes.

A FLNC pathogenic variant was identified in 28 patients corresponding to a prevalence ranging from 1 to $8 \%$ depending on the cardiomyopathy subtype. Truncating variants were always identified in patients with dilated cardiomyopathy, while missense or in-frame variants were found in other phenotypes. A personal or family history of sudden cardiac death (SCD) was significantly higher in patients with truncating variants than in patients carrying missense variants $(p=0.01)$. This work reported for the first time a left ventricular non-compaction associated with FLNC pathogenic variant.

This work highlights the role of FLNC in cardiomyopathies. A correlation between the nature of the variant and the cardiomyopathy subtype was observed as well as with SCD risk.

Key Word: Genotype-phenotype correlation, FLNC, cardiomyopathies, next generation sequencing, myopathy, 


\section{Introduction:}

Filamin $C$ is a homodimeric protein encoded by FLNC gene (7q32) containing 47 coding exons (NM_001458.4). Pathogenic variants in the FLNC gene have been firstly reported to cause dominant myofibrillar and distal myopathy ${ }^{1}$. More recently, dominant pathogenic variants in FLNC have also been linked to the development of isolated cardiac phenotypes, including hypertrophic cardiomyopathies (HCM $)^{2}$, restrictive cardiomyopathies $(\mathrm{RCM})^{3}$ and dilated cardiomyopathy $(\mathrm{DCM})^{4}$. In non-compaction left ventricle cardiomyopathy, (LVNC), a FLNC variant was recently involved but associated with a second variant in RYR2 gene 5 .

Functionally, filamin C is a striated muscle protein that crosslinks sarcoplasmic F-actin and anchors the cell membrane through the sarcoglycan complex with the cytoskeleton and the sarcomere Z-disk6. The protein is composed of an actin binding domain, 24 Immunoglobulin (Ig) domains divided into ROD1 and ROD2 sub-domains and a C-terminal dimerization domain ${ }^{7}$. Filamin $\mathrm{C}$ plays a role in the myofibril maintenance and the myogenesis in cardiac and skeletal muscles ${ }^{8}$. It has many interacting partners at the sarcomere Zdisk such as nexilin, actinine, myopodin and myozenin ${ }^{9}$ which confer a role in the sarcomere maintenance and repair after myofibrillar damage ${ }^{10}$.

It appears from recent publications that some variants are preferentially associated with myopathies and others with cardiomyopathies 1,2,11. Only few publications on cohorts with FLNC variants are published and the pathophysiology of FLNC-related cardiomyopathy is poorly understood. The prevalence of this gene in cardiomyopathies, the nature and location of variants in the protein, as well as their impact on the phenotype are not yet studied. This makes difficult the interpretation of variants and their impact as disease causing on the sub-phenotype of cardiomyopathies. The objective of this retrospective study is to establish the prevalence of pathogenic variants in the FLNC gene in the different subtypes of cardiomyopathies and to search for genotype-phenotype associations in the perspective of improving genetic diagnosis and counseling as well as early cardiac management and primary prevention.

\section{Materiel and Methods}

\section{Patients and inclusion criteria}


In the context of the molecular diagnosis of cardiomyopathies, in the functional unit of Cardiogenetics and Myogenetics at the Pitié-Salpêtrière hospital between 2010 and 2017, a total of 1150 unrelated index patients including 700 hypertrophic cardiomyopathy (HCM), 300 dilated cardiomyopathy (DCM), 50 restrictive cardiomyopathy (RCM) and 100 left ventricle non-compaction (LVNC) patients have been sequenced on a custom panel of 51 cardiomyopathy disease-causing genes, including FLNC. According to our molecular strategy, most of HCM and DCM patients were first analyzed on a small panel of major genes (MYH7, MYBPC3, TNNT2, TNNI3, and MYL2, +/- LMNA gene in case of DCM) before being sequenced on the extensive panel (Figure 1). This restricted panel allowed the molecular diagnosis in $30 \%$ of $\mathrm{HCM}$ and $10 \%$ of $\mathrm{DCM}$ and avoided sequencing some of them, particularly in $\mathrm{HCM}$, on the large panel of genes. Genetic screening was done after all the patients have signed an informed written consent for genetic analysis according to the French legislation. Twenty-eight index-patients presenting a unique disease-causing variant of FLNC were selected for the rest of the study.

The clinical diagnosis of cardiomyopathy has been established by electrocardiography, echography, and MRI according to usual European recommendations in 14 expert centers in France ${ }^{12-15}$.

\section{Sequencing}

FLNC gene was sequenced in the context of the molecular diagnosis of inherited cardiomyopathies using a targeted custom panel of 51 genes. The panel $(256 \mathrm{~kb})$ includes all coding regions and flanking intronic regions (+/- $20 \mathrm{bp})$ of genes responsible of the diverse morphological subtypes of cardiomyopathies (HCM, DCM, RCM, LVNC and arrhythmogenic right ventricle cardiomyopathies (ARVC)), pediatric phenotypes as well as some cardiomyopathies due to metabolic disorders and/or syndromic cardiomyopathies (LAMP2, PRKAG2, GLA) (list of genes in supplementary data).

Patients' DNAs were extracted from peripheral blood with Qiasymphony® (Qiagen, Hilden, Germany) and qualitatively checked using Tape Station DNA genomic array(Agilent, Santa Clara, USA). Custom targeted gene enrichment and DNA library preparation were performed using the NimbleGen EZ choice probes $®$ and Kappa Library preparation kit according to the manufacturer's instructions (Nimblegen $®$, Roche Diagnostics, Madison, USA).The targeted regions were sequenced using the Illumina MiSeq platform on a 500 cycle Flow Cell (Illumina, Santa Cruz, USA) and MiSeq Software generates FASTQ format files after demultiplexing patients' sequences. Merged single reads and paired-end reads were then aligned on $\mathrm{Hg} 19$ human reference genome, using BWA-MEM. Variant calling was performed using the GATK Haplotype Caller programthen annotated using ANNOVAR. 


\section{Variant's interpretation}

Pathogenicity of variants was determined according to current ACMG guidelines ${ }^{16}$ that recommend classifying variants into 5 categories: (1) pathogenic, (2) likely pathogenic, (3) uncertain significance (VUS), (4) likely benign, or (5) benign. A recent publication dedicated to cardiomyopathies recommended the use of a frequency threshold of $0.01 \% 17$. We evaluated each variant considering several parameters: (i) a frequency threshold $<0.01 \%$ in GnomAD database (URL: http://gnomad.broadinstitute.org/), (ii) the insilico prediction from multiple algorithms (Polyphen2, SIFT, GVGD and Mutation Taster for missense variants and SpliceSiteFinder like $®$, MaxEntScan $®$, NNSPLICE $®$, GeneSplicer ${ }^{\circledR}$ and Human Splicing Finder ${ }^{\circledR}$ for splicing variants), (iii) the location of the variant in the gene and the resulting protein, (iv) a careful review of the literature (HGMD Pro and Pubmed review), (v) functional studies and segregation analyses when available. Additionally, we looked at a personal database of pathogenic variants related to our experience on the molecular diagnosis of cardiomyopathies which could help to confirm the pathogenicity (frequency, familial analyses). In practice, we considered as "pathogenic", a variant with confirmed pathogenicity criteria and already proved as responsible for cardiomyopathies (published with proofs of pathogenicity or functional studies, personal database with segregation analysis) or a novel truncating variant (non-sens, frameshift or splice variant), with a frequency below $0.01 \%$ in GnomAD. We considered as "likely pathogenic", unpublished missense variants with a frequency below $0.01 \%$ and unknown in our database, located in a functional domain of the protein and with pathogenicity prediction tools mainly (at least 3 out of 4 tools) in favor to a strong effect. Pathogenicity of this likely pathogenic variant is also supported by an informative segregation analysis. Unpublished missense variants with a frequency below $0.01 \%$, unknown in our database, located in a functional domain of the protein and with pathogenicity prediction tools mainly in favor to a strong effect but without segregation analysis available were considered as VUS favor pathogenic. Variants of unknown significance were new missense variants with no evidence for in silico predicted deleteriousness and published variants with a frequency over $0.01 \%$.

Detection of copy number variation (CNVs) was performed, after coverage normalization, by computing the ratio of a target's coverage of a given individual over the mean coverage of this target across all patients of the same sequencing run. Their putative frequency was checked with DGV (Database of genomic variants), which collect all large gene rearrangements in a normal population.

Only variants interpreted as certainly pathogenic, likely pathogenic and VUS favor pathogenic were considered for further clinical study and were confirmed by Sanger sequencing on the initial DNA sample. 
Statistical analysis was performed using Student or Fisher test when adapted.

\section{Results:}

\section{Clinical pattern}

Twenty-eight index patients carrying a probably or certainly pathogenic FLNC disease-causing variants were retained for further studies. Among them, 13 were affected with $\mathrm{HCM}, 10$ with $\mathrm{DCM}, 4$ with RCM and 1 by LVNC (Table 1). No clinical skeletal myopathy was noted in these patients and CK levels were normal when available (14/28 patients). Twenty-three patients were Caucasian, 3 from Africa, 1 from Asia and 1 from north Africa.

The $13 \mathrm{HCM}$ patients comprised 10 familial and 3 sporadic patients. Mean age at diagnosis was 33 years (SD 20.4 years), and sex ratio was 5 women for 8 men. Mean interventricular septum thickness was $15.9 \pm 3.5 \mathrm{~mm}$, mean posterior wall thickness was $15.2 \pm 5.4 \mathrm{~mm}$, and mean maximal wall thickness was $16.7 \pm 2.5 \mathrm{~mm}$. For all patients the ejection fraction of left ventricle was normal. Patient 12 presented an atypical HCM, with maximum wall thickness of $18 \mathrm{~mm}$.preserving the interventricular septum at $11 \mathrm{~mm}$ and a normal systolic function leading to the diagnosis of $\mathrm{HCM}$ with a restrictive profile. However, the patient had a congestive heart failure and there were two relatives with similarly, a HCM with restrictive profile requiring heart transplant. Probably the phenotype in the family a mixed phenotype of HCM and RCM. Regarding ECG parameters in the 13 mutated patients, atrial fibrillation was observed in 3 patients, atrioventricular block degree 1 was observed in 2 patients. Complete branch block was observed in 2 patients with HCM. Two patients have been implanted with adefibrillator. Familial history of sudden death before 50 years old was reported in 2 families but no index patient of the cohort deceased froma cardiovascular cause.

In the group of DCM patients $(\mathrm{N}=10)$, we reported 6 familial and 4 sporadic cases with a sex ratio of 6 females and 4 males. Mean age at diagnosis of 31 years (SD: 13.7 years). The mean LVEF was $33.6 \pm$ 9.5\%. On ECG, two patients presented a moderate short interval PR, two others presented a complete left bundle block branch, one patient presented AF and three had non sustained ventricular tachyarrhythmia (nsTV), no patient presented an AVB. Duringthe follow up, three patients have been hospitalized due to acute heart failure, and three patients suffered from sudden cardiac death (including one patient with confirmed ventricular tachycardia). For patients who died from inaugural sudden cardiac death, data were not fully available. 
In the 4 patients with RCM, mean age at diagnosis was 36 years (SD 9.9 years), and sex ratio of 1:1. No significant AVB was observed whereas atrial fibrillationwas observed inallpatientsand ICD was implanted in two patients. No SCD was observed but one patientdeceasedbecause of heart failure (while waiting for heart transplant) at 36 years old, one still alive patient underwent a heart transplantation at 36 years old and one additional patient is still waiting for heart transplant.

Finally, the patient with LVNC was diagnosed at 36 years old, LV EF was $49 \%$, he presented complete right branch block and was implanted with a defibrillator (primary prevention due to nsVT). This case was reported to be sporadic. In this patient abnormal trabeculations on echocardiography and on MRI and criteria were present (on MRI and end-diastolic measurement: non compacted/compacted ratio was 4.2) leading to the diagnosis LVNC.

When considering the whole cohort, 9 patients had atrial fibrillation $(32 \%)$ and 5 patients had conduction defects including 2 patients with AVB degree 1 and 3 patients with relatively short PR. Five patients had non sustained VT (17.8\%) including 2 DCM patients who died from SCD. In 7 cases (25\%), sudden cardiac death before 50 years has been reported in family history. Nine patients (32\%) have been implanted with automatic defibrillators in primary prevention. Acute heart failure was observed in 7 patients (25\%) including one patient was transplanted at 36 years old. Four patients died of cardiac cause including 3 from SCD and 1 from heart failure.

\section{Genetic results:}

Prevalence of FLNC disease-causing variants in cardiomyopathies:

The overall prevalence of patients carrying a unique FLNC pathogenic variant in our cohort was evaluated from 1 to $8 \%$ according to cardiomyopathy subtypes, distributed as following; $1.3 \%$ in $\mathrm{HCM}, 3 \%$ in $\mathrm{DCM}$, $8 \%$ in RCM and $1 \%$ in LVNC.

\section{Molecular genetics}

Among the 28 index cases with a pathogenic variant in FLNC, 20 index patients from all phenotypes presented a family history with affected relatives, suggesting an autosomal dominant pattern of inheritance and 8 patients without family history were considered as sporadic cases. In ten index cases, we could analyze genetic status of relatives. In RCM, 4 relatives of patients 26 and 27 ; in DCM, 5 relatives from the 5 families; and in HCM, 3 relatives from 3 families in which segregation analysis was performed are represented in Figure 2. In these families, 10 relatives carrying the variant harbored the same 
cardiomyopathy phenotype as the proband. In two DCM families, 2 relatives were presymptomatic carriers but genotyped at a younger age than the age of onset of the disease in the index case (relatives of patient 4 and patient 6) (details in Fig. 2).

The spectrum of $F L N C$ variants showed the identification of 28 unique disease-causing variants including 27 novel ones never published before (Table 1 and 2). Among these variants and according to the FLNC reference sequence (NM_001458.4), there are 10 null variants consisting in 3 nonsense (stop) variants (p.Tyr928*, p.Cys2555* and p.GIn2549*), 2 intronic variants abolishing the splicing of mRNA (c. $1412-1 \mathrm{G}>\mathrm{A}$ and $\mathrm{c} .601+1 \mathrm{G}>\mathrm{T}), 5$ duplications or deletions leading to a shift in the reading frame (p.Tyr7Thrfs*51, p.Glu238Argfs*14, p.lle683Argfs*9, p.Val1198Glyfs*64, p.Tyr2373Cysfs*7), one in frame deletion (p.Pro2643_Leu2645del) and one in frame duplication (p.lle1946_Thr1947dup), and 16 missense variants identified by the residue change (p.Ser1194Leu, p.Gly1424Val, p.Ser1624Leu, p.Ile1666Thr, p.Gly2011Glu, p.Gly2039Arg, p.Arg2140GIn, p.Val2297Met, p.Pro2298Leu, p.Gly2299Ser, p.Arg2318Trp, p.lle2359Thr, p.Val2375Leu, p.Arg2410Cys, p.GIn2417Pro, and p.Arg2495His).

As the nonsense variants were spanning all along the gene (regions corresponding to actin binding domain, ROD1 and ROD2 domains), missense variants were clustered in the ROD1 and ROD2 domains. The two in-frame deletion and duplication were respectively located in the dimerization domain and the ROD2 domain (Figure 3).

A relation between the genotype and the phenotype was observed both regarding the nature of the variant and its localization in the gene or protein domains. Regarding the mechanism of the mutation, all 10 patients presenting with a dilated cardiomyopathy were carriers of a truncating variant while all 13 patients presenting with $\mathrm{HCM}$ carried missense variants ( $\mathrm{p}$ value; 7 10-8)(Fig 3). In the 4 patients with restrictive cardiomyopathy, two patients had missense variants; one had an in-frame duplication and the other an inframe deletion and the only patient with LVNC carried a missense variant. Regarding the risk for SCD, a personal or a familial history of SCD was reported in $7 / 10$ patients with truncating variants (70\%) and in $3 / 16$ patients $(19 \%)$ carrying a missense variants $(p=0.01$, Fisher Exact Test).

Regarding the variant localization and the subtype of cardiomyopathy: in HCM patients, missense variants are clustered in ROD2 domains (10 variants) then in ROD1 (3 variants). In the 4 patients presenting RCM, 3 variants were located in ROD2 domain, particularly clustered on residue 2297 and 2298, and one in the dimerization domain. The LVNC associated variant was located in ROD2 domain. In patients with DCM, as all variants were null variants disrupting the reading frame, the position of the variant has probably limited 
impact, the likely consequence being mRNA decay of the mutated allele. Additionally, no particular association could be observed regarding the rhythm disturbance and the position of the variant in the protein. Nevertheless, in the 9 patients who were implanted with ICD, 7 were mutated in the ROD2 domain. In two patients with restrictive cardiomyopathy, transplanted or awaiting for transplantation, the variants were adjacent, both located in exon 41 (c.6889G>A_p.Val2297Met, and c.6893C>T_p.Pro2298Leu) in the Ig domain 20.

\section{Discussion}

This study was focused on FLNC, a gene recently involved in isolated cardiomyopathies. The particularity of our study is to involve all subtypes of cardiomyopathies as well as the analysis of all types of variants (missense and truncating). This cohort of 1150 patients provides new findings regarding the prevalence of this mutated gene in different subtypes of cardiomyopathies as well as some phenotype-genotype relations.

\section{Prevalence}

In our cohort composed of various cardiomyopathies, the estimated prevalence of patients carrying a unique FLNC variants were ranging from $1 \%$ to $8 \%$ according to cardiomyopathy subtypes, with about $1-3 \%$ in HCM and DCM. In a recent published data ${ }^{11}$, in which only truncated variants were considered, the estimated prevalence of FLNC was about $3.9 \%$ ( $n=508$ patients) in DCM, and $3 \%$ in arrhythmogenic cardiomyopathies ( $n=219$ patients) and $2.2 \%$ in RCM (1/45). These observations lead to consider FLNC gene as a gene especially prevalent in the $\mathrm{DCM}^{18}$. In another publication dedicated to hypertrophic cardiomyopathy, the estimated prevalence on 92 patients was $7.6 \%$ and comprised 1 truncating variant and 6 missense variants ${ }^{2}$. These results are in accordance with ours for DCM phenotype, nevertheless the prevalence in RCM patient is higher in our cohort. It could be explained by the fact that we considered truncating as well as non-truncating especially in frame insertion and deletion variants. This relatively high prevalence of $F L N C$ in $\mathrm{RCM}(8 \%)$ is a novel finding that extend the spectrum of genes in this particularly severe phenotype. In HCM, the prevalence found in our cohort is lower; this could be due to the size or characteristics of the cohort. In LVNC, this is the first time that a unique pathogenic variant in FLNC was found as disease-causing as in the only previous published patient with LVNC, the FLNC variant was associated with a RYR2 variant 5 .

Genotype Phenotype correlation 
We searched first a putative relation between the subtype of cardiomyopathy and the mechanism of the mutation and its localization. In our cohort, all HCM patients were carriers of a missense variant, and all DCM patients carried a truncating variant leading to haploinsufficiency. Our results emphasize a strong correlation between the subtypes of cardiomyopathy and the nature of disease causing variant. As compared with the literature, upon the 59 published variants consistent with our criteria for pathogenicity, 14 variants were associated with a myopathy phenotype (6 in distal myopathy and 8 in myofibrillar myopathy $)^{1}$ and 45 were associated with an isolated cardiomyopathy (24 variants in DCM, 19 in HCM, 2 in $\mathrm{RCM}$ ). Among all these published variants, 28 were truncating variants, 29 missense variants, and 2 were deletion or insertion without shift of the reading frame. For this study, we used stringent criteria for classification of variants. Nevertheless, it is important to note that some discrepancy could exist between studies, particularly in gene variants interpretation, especially for missense variants. Similarly to our observation, in HCM patients, among the 19 published variants, 18 were missense variants. Regarding the localization of missense variants in the protein domains, we observed a larger proportion of variants located in the ROD2 domain in HCM patients which is in accordance with published data that revealed a cluster of missense variants in ROD2 domain of filamin in HCM phenotype $2,11,19$. This region is the interaction domain between filamin $\mathrm{C}$ and proteins of the Z-disk, as synaptopodin, myozenin, and nexilin and could be an interesting way to understand the mechanism of pathogenicity of these missense variants. The pathophysiological mechanism of missense variants was explored in rat fetal cardiomyocytes and showed that variants located in actin binding domain and dimerization domain lead to filamin aggregates ${ }^{10}$. In this case, the hypothesis was a dominant negative model, however the pathophysiology of this aggregate and the link to HCM development remain unclear. A discrepancy observed, is the variant p.Ser1624Leu identified in an HCM patient in our cohort and in a RCM patient in the publication of Brodhel et al. The patient included in this cohort had a diagnosis of HCM since MWT was $18 \mathrm{~mm}$ and normal systolic function. However the patient had a congestive heart failure and there were two relatives with a cardiomyopathy requiring heart transplant. Probably the phenotype in the family a mixed phenotype of HCM and RCM, which presents similarities with the previously published family with (pure) RCM (since no LV hypertrophy was noticed in this publication).

Additionally, in a previous cohort, Valde's-Mas et al, 2014 found that $34 \%$ of the HCM patients carriers of pathogenic variant in FLNC had elevated levels of serum creatine kinase (CK). In this work, all patients have a normal CK level. When presenting a cardiomyopathy, patients were systematically checked for muscle symptoms. A muscular RMI, which could help to detect infra-clinic myopathy, was not systematically performed in this cohort. Two patients presented a transitory CK elevation (patient 21 et 27) but RMI didn't 
find any muscle sign. The difference between the cohort of Valde's-Mas et al is difficult to explain and could be a difference in the cohort recruitment.

However, the implication of missense variants in HCM is questioned and need to be confirmed. Mainly, two publications didn't detect significant excess of rare missense variants between HCM cases and controls 20,21. One limitation discussed by Walsh is an insufficient size of the cohort ${ }^{21}$. Additionally, in the publication of Cui et al., at least three differences could be noticed: (1) the ethnic origin of the cohort, (2) the threshold of variant frequency and (3) the fact that $33 \%$ of their patients were carriers of a second mutation in a major gene which could induce a bias in the expression of the disease 20.

Among the 24 variants reported in DCM, 23 are truncating variants and the only missense variant found was associated with a null variant in a neonatal case of $\mathrm{DCM}^{22}$. In this case, the congenital form of the DCM could be due to the cumulative effect of the missense variant associated with the heteroallelic truncating variant in FLNC. These finding reinforce the role of FLNC null variants in the development of DCM. The pathophysiology of the truncating variants and the development of DCM was explored in zebrafish models and concluded to a haploinsufficiency model ${ }^{4}$. Similarly, in myopathies, the haploinsufficiency mechanism and the RNA decay activation have been confirmed on fish model ${ }^{8}$. In RCM and LVNC cardiomyopathies 3,5 , no correlation could be observed due to a small number of patients published. However, it was interesting to notice that, additionally to missense variants, the only variants consisting in in-frame insertion or deletion were associated with RCM.

Secondly, we searched a relationship between the variant type and the arrhythmia pattern. In our cohort, $32 \%$ of patients were ICD implanted and a familial history of SCD was observed in $25 \%$ of cases. A familial or a personal history of SCD was significantly more frequently observed in patients carrying truncating variants than in patients carrying a missense variant suggesting that null or truncating variants may predispose to severe arrhythmias. Additionally, in this cohort, a high number of patients presented a conduction defect (Table 1) respectively $30 \%$ in $\mathrm{HCM}$ and $40 \%$ in DCM suggesting that the mechanism of the variant has no important impact on this clinical feature. In a recent publication on arrhythmogenic cardiomyopathies, it was observed a conduction defect in $12 \%$ of the patients, sudden death in $15 \%$ of the patients and appropriate ICD Shock in $10 \%{ }^{11}$. These finding highlighted the arrhythmogenic pattern of FLNC truncating variants cardiomyopathies. Up to now, no large cohort of FLNC related cardiomyopathies with a long term follow up has been published, but the initial data in several independent cohorts (this work 
and reference 16) support the high risk of SD and conduction defect, that should be carefully evaluated in primary prevention of cardiac death.

\section{Limitations:}

This work details the phenotype of limited number of index cases carrying a unique pathogenic variant in FLNC gene. Thus, regarding to the penetrance, it seems that the age of onset of all affected patients may varies from young age to adult age. Additionally, the total number of carriers is too small to conclude to the penetrance of theses variants.

In conclusion, we presented in this study the results of the sequencing of a targeted panel of cardiomyopathy genes in a large cohort of index patients with different subtypes of cardiomyopathies. This allowed to evaluate the prevalence of $F L N C$ variants in these different groups and then, in mutated patients to exhibit some genotype-phenotype relations. For the first time, a pathogenic variant in FLNC was associated with LVNC phenotype. We observed a relation between the nature of the variant and the subtype of cardiomyopathy as well as a clear arrhythmogenic pattern in patients with truncating variants. Our results therefore support the emerging role of FLNC in isolated cardiomyopathies and the inclusion of this gene in panels dedicated to sequencing of patients with a cardiomyopathy, whatever the subtype. This data should be taken in consideration for genetic counseling in patient's families.

\section{Bibliography}

1. Fürst DO, Goldfarb LG, Kley RA, Vorgerd M, Olivé M, van der Ven PF. Filamin C-related myopathies: pathology and mechanisms. Acta Neuropathol. 2013;125(1):33-46.

2. Valdés-Mas R, Gutiérrez-Fernández A, Gómez J, et al. Mutations in filamin C cause a new form of familial hypertrophic cardiomyopathy. Nat Commun. 2014;5:5326.

3. Brodehl A, Ferrier RA, Hamilton SJ, et al. Mutations in FLNC are Associated with Familial Restrictive Cardiomyopathy. Hum Mutat. 2016;37(3):269-279.

4. Begay RL, Tharp CA, Martin A, et al. FLNC Gene Splice Mutations Cause Dilated Cardiomyopathy. JACC Basic Transl Sci. 2016;1(5):344-359.

5. Miszalski-Jamka K, Jefferies JL, Mazur W, et al. Novel Genetic Triggers and Genotype-Phenotype Correlations in Patients With Left Ventricular Noncompaction. Circ Cardiovasc Genet. 2017;10(4).

6. Thompson TG, Chan YM, Hack AA, et al. Filamin 2 (FLN2): A muscle-specific sarcoglycan interacting protein. J Cell Biol. 2000;148(1):115-126.

7. van der Flier A, Sonnenberg A. Structural and functional aspects of filamins. Biochim Biophys Acta. 2001;1538(2-3):99-117.

8. Fujita $M$, Mitsuhashi $\mathrm{H}$, Isogai $\mathrm{S}$, et al. Filamin $\mathrm{C}$ plays an essential role in the maintenance of the structural integrity of cardiac and skeletal muscles, revealed by the medaka mutant zacro. Dev Biol. 2012;361(1):79-89.

9. Takada F, Vander Woude DL, Tong HQ, et al. Myozenin: an alpha-actinin- and gamma-filaminbinding protein of skeletal muscle Z lines. Proc Natl Acad Sci U S A. 2001;98(4):1595-1600.

10. Leber $Y$, Ruparelia AA, Kirfel $G$, et al. Filamin $C$ is a highly dynamic protein associated with fast repair of myofibrillar microdamage. Hum Mol Genet. 2016;25(13):2776-2788. 
11. Ortiz-Genga MF, Cuenca S, Dal Ferro M, et al. Truncating FLNC Mutations Are Associated With High-Risk Dilated and Arrhythmogenic Cardiomyopathies. J Am Coll Cardiol. 2016;68(22): 2440-2451.

12. Elliott PM, Anastasakis A, Borger MA, et al. 2014 ESC Guidelines on diagnosis and management of hypertrophic cardiomyopathy: the Task Force for the Diagnosis and Management of Hypertrophic Cardiomyopathy of the European Society of Cardiology (ESC). Eur Heart J. 2014;35(39):2733-2779.

13. Rammos A, Meladinis V, Vovas G, Patsouras D. Restrictive Cardiomyopathies: The Importance of Noninvasive Cardiac Imaging Modalities in Diagnosis and Treatment-A Systematic Review. Radiol Res Pract. 2017;2017:2874902.

14. Jenni R, Oechslin E, Schneider J, Attenhofer Jost C, Kaufmann PA. Echocardiographic and pathoanatomical characteristics of isolated left ventricular non-compaction: a step towards classification as a distinct cardiomyopathy. Heart. 2001;86(6):666-671.

15. Pinto YM, Elliott PM, Arbustini E, et al. Proposal for a revised definition of dilated cardiomyopathy, hypokinetic non-dilated cardiomyopathy, and its implications for clinical practice: a position statement of the ESC working group on myocardial and pericardial diseases. Eur Heart J. 2016;37(23):1850-1858.

16. Richards S, Aziz N, Bale S, et al. Standards and guidelines for the interpretation of sequence variants: a joint consensus recommendation of the American College of Medical Genetics and Genomics and the Association for Molecular Pathology. Genet Med. 2015;17(5):405-424.

17. Walsh R, Thomson KL, Ware JS, et al. Reassessment of Mendelian gene pathogenicity using 7,855 cardiomyopathy cases and 60,706 reference samples. Genet Med. 2017;19(2):192-203.

18. Pérez-Serra A, Toro R, Sarquella-Brugada G, et al. Genetic basis of dilated cardiomyopathy. Int J Cardiol. 2016;224:461-472.

19. Gómez J, Lorca R, Reguero JR, et al. Screening of the Filamin C Gene in a Large Cohort of Hypertrophic Cardiomyopathy Patients. Circ Cardiovasc Genet. 2017;10(2).

20. Cui H, Wang J, Zhang C, et al. Mutation profile of FLNC gene and its prognostic relevance in patients with hypertrophic cardiomyopathy. Mol Genet Genomic Med. 2018;6(6):1104-1113.

21. Walsh R, Mazzarotto F, Whiffin N, et al. Quantitative approaches to variant classification increase the yield and precision of genetic testing in Mendelian diseases: the case of hypertrophic cardiomyopathy. Genome Med. 2019;11(1):5.

22. Reinstein E, Gutierrez-Fernandez A, Tzur S, et al. Congenital dilated cardiomyopathy caused by biallelic mutations in Filamin C. Eur J Hum Genet. 2016;24(12):1792-1796.

23: Janin A, N'Guyen K, Habib G, et al. Truncating mutations on myofibrillar myopathies causing genes as prevalent molecular explanations on patients with dilated cardiomyopathy. Clin Genet. 2017 Dec; 92(6):616-623.

24: Tucker NR, McLellan MA, Hu D, et al. Novel Mutation in FLNC (Filamin C) Causes Familial Restrictive

Cardiomyopathy.Circ Cardiovasc Genet. 2017 Dec;10(6).

25: Schubert J, Tariq M, Geddes G, et al.Novel pathogenic variants in filamin C identified in pediatric restrictive cardiomyopathy. Hum Mutat. 2018 Dec;39(12):2083-2096.

\section{Legends to Figures:}

Figure 1: Flow chart describing the genetic molecular testing depending of the cardiomyopathy phenotype.

Figure 2: Pedigree tree of 4 families with FLNC pathogenic variants for which genetic segregation was possible. Squares indicate males, circles indicate females, slashes indicate deceased individuals, black shading indicates a dilated cardiomyopathy (DCM) phenotype. The arrows indicate the proband.

Heterozygous carriers (+/-) and non-carriers (-) of a FLNC variant are indicated. 
Figure 3: Distribution of FLNC variants along the protein in various subtypes of cardiomyopathies according to their nature and location. 